IV Congreso Chileno de Antropología. Colegio de Antropólogos de Chile A. G, Santiago de Chile, 2001.

\title{
Cuidadores Formales en la Institucionalización de Larga Estadía.
}

\section{María Belén Berruti. y Mariana Buzeki.}

Cita:

María Belén Berruti. y Mariana Buzeki. (2001). Cuidadores Formales en la Institucionalización de Larga Estadía. IV Congreso Chileno de Antropología. Colegio de Antropólogos de Chile A. G, Santiago de Chile.

Dirección estable: https://www.aacademica.org/iv.congreso.chileno.de.antropologia/65 ARK: https://n2t.net/ark:/13683/ef8V/z8m 


\title{
Cuidadores Formales en la Institucionalización de Larga Estadia
}

\author{
María Belén Berruti y Mariana Buzeki
}

\section{Introducción}

El desarrollo científico-técnico y los cambios culturales a lo largo del siglo han producido por un lado el alargamiento de la expectativa de vida y por otro un mayor control de la natalidad. De esta manera asistimos a un acelerado y progresivo proceso de envejecimiento poblacional, expresado en datos censales de nuestro país en una proporción de personas mayores de 60 años del $13 \%$ del total de habitantes en 1991, a diferencia del $7 \%$ en el que representaba este conjunto poblacional en 1950. Dentro de dicho conjunto la población mayor de 75 años constituía el $25,4 \%$ en 1991 , señalando una tendencia marcada de crecimiento en esta franja que se denomina "envejecimiento del envejecimiento" (Oddone, 1998).

En la ciudad de Mar del Plata un $16 \%$ de la población es mayor de sesenta años, constituyendo un factor explicativo del $44 \%$ de aumento de esta franja en la década del ' 80 , la migración constante de jubilados hacia nuestra ciudad, comparten expectativas de bienestar y ocio recreativo promovidas por un imaginario que ha influído fuertemente en la elección del lugar de residencia (Berruti, Buzeki, de los Reyes, Roose, 1999) No existen en nuestro país, en comparación con los países desarrollados, políticas sociales que contemplan los problemas del envejecimiento a pesar de los esfuerzos de diferentes sectores de la sociedad. En este contexto se observa el crecimiento de la institucionalización de ancianos debido a la alta incidencia de demencias y de diferentes grados de deterioro físico y cognitivo en esta franja etaria. Otros factores estructurales que posibilitan el crecimiento de instituciones geriátricas son los cambios producidos en la estructura y roles familiares, la reducción espacial de las viviendas y la inexistencia o insuficiencia de alternativas a la institucionalización geriátrica. Esta situación es desatendida por el Estado lo que provoca la proliferación de geriátricos privados en nuestra ciudad con escaso control por parte de aquel.
Según registros de febrero de 1999 del Área Tercera Edad de la Subsecretaría del Menor, la Familia, Discapacitados y la Tercera Edad de la Municipalidad de General Pueyrredon, existen en Mar del Plata cincuenta y tres establecimientos geriátricos habilitados. La expansión de la actividad ha originado paralelamente en los últimos años el funcionamiento de cuarenta y ocho pequeños hogares, habilitados por la autoridad comunal en casas de familia. Con una oferta estimada de 2600 plazas entre los dos tipos de establecimientos, la proporción de internación geriátrica sobre la población mayor de 60 años es de un $2 \%$, mientras que en el país este porcentaje se calcula en un $1,5 \%$, considerándose bajo en comparación con otros países. En cuanto a la evolución de la capacidad habilitada en la ciudad para este tipo de internaciones, se constata un aumento importante en los últimos trece años: 1200 camas desde 1985 hasta fines de 1998.

El presente trabajo intenta describir y explicar el modo en que los cuidadores formales conciben la práctica de cuidado a ancianos dependientes en instituciones de larga estadía a partir del análisis del discurso de los mismos.

La metodología a emplear es cualitativa y el diseño es exploratorio-descriptivo. Como técnicas de recolección de datos se utilizaran serán entrevistas semiestructuradas a propietarios de instituciones geriátricas. Se analizaran los contenidos de las entrevistas.

La cantidad de entrevistas seleccionada se basará en la estrategia del muestreo teórico, según la cual se irian agregando casos a medida que se requieran nuevas intelecciones o refinamientos de las ya alcanzadas por el investigador. Esta estrategia, basada en la inducción analítica, hace posible que la recolección de datos se realice en forma simultánea al análisis de contenidos. Los casos adicionales permiten comprobar si los descubrimientos realizados entre determinados sujetos son aplicables a otros con características y situaciones diferentes (Taylor y Bogdan, 1986). 


\section{La internación geriátrica}

Partimos de la idea que la internación geriátrica de larga estadía (ILE) constituye un proceso en cuya construcción participan diversos actores sociales. Entre ellos encontramos a los ancianos internados, sus familiares directos e indirectos (cuidadores familiares que constituyen el apoyo informal), los profesionales y cuidadores del ámbito del geriátrico y por último, los dueños y encargados de las instituciones de internación geriátrica ,quienes conforman el aspecto formal de los cuidados (Berruti, 2001).

Se asume que el fenómeno de la ILE solo es comprensible partiendo de las diferentes perspectivas de estos actores involucrados en su construcción. Es por ello que como investigadores intentamos aproximarnos a la subjetividad de los distintos participantes buscando reconstruir cada perspectiva, partiendo de la idea de que hallaremos la comprensión del fenómeno, en el entrecruzamiento de las mismas.

Con el término cuidador se alude a aquella persona que asiste o cuida a otra afectada de cualquier tipo de discapacidad, minusvalía o incapacidad que le dificulta o impide el desarrollo normal de sus actividades vitales 0 de sus relaciones sociales (Flórez Lozano et al, 1997) El proceso de cuidar implica una relación interpersonal, que se caracteriza por la asimetría, es una acción que apunta a ayudar a reconstruir la autonomía del sujeto vulnerable, implica no solo ayudar a la persona enferma sino también a su entorno. Para ello es necesario informar, dar la mayor cantidad de conocimiento para que la persona y/ o su entorno puedan decidir a lo largo de este proceso. Cuidar de alguien es ayudarle a ser, a ser en su singularidad y especificidad (Torralba i Roselló, 1998).

\section{La perspectiva de los cuidadores formales: los propietarios}

En este apartado nos centraremos en un tipo de cuidador formal especial y que resulta central en el cuidado de los ancianos. Se trata de los propietarios de establecimientos geriátricos privados de nuestra ciudad. Los temas que aparecen en las entrevistas se pueden dividir en tres aspectos: los aspectos referidos a la población incluyendo en ella a los propios ancianos y sus familiares, los aspectos referidos a la organización, y por último los aspectos personales o idiosincrásicos. En cuanto al primer aspecto observamos la aparición de categorías que definen el tipo de relación que los propietarios y /o encargados establecen con los ancianos y familiares.

En el discurso de los propietarios se expresa una tendencia a la masificación de los ancianos, a la despersonalización, pensando al trato igualitario como al ideal de la atención. Es así como se cpnfiguran prácticas de cuidado que terminan cosificando a los individuos al negar las diferencias entre ellos.

(...) "bueno todo lo que se le debe dar al abuelo, peluquería, manicura, bueno todo, todo lo que se le debe dar al abuelo, eso lo hago porque es como si tuviera mis 36 hijos" (...)el abuelo es algo muy lindo...es muy apasionante manejarlos a ellos, es hermosísimo.....() (...) "yo compro la colonia, no porque me sobre, sino porque si a vos te traen colonia y a Juancita no se la traen alguien se la tiene que traer, entonces yo compro la misma colonia que usa una abuela, ponele que sea Heno de Pravia, yo compro Heno de Pravia para todos"(...)

(...)"son una criatura yo le doy un beso primero a uno y después al otro, y si le doy el diario primero a uno mañana le tengo que dar al otro, porque son celosos" (...). Al homogeneizar a la población mediante un trato igualitario el encargado puede establecer un control mucho mayor sobre ella, evitando conflictos propios de las relaciones entre individuos diferentes. De esta manera se propicia un vínculo de dependencia aumentando por un lado su propia autoridad ante el anciano y por ende ante su familia y por otro aumentando el estado de dependencia que dio origen a la internación.

La generación de vínculos de dependencia es vivida muchas veces con hostilidad por ambos lados ya que esto genera la necesidad de aumentar los cuidados por parte de la institución ya que se incrementan las demandas de los ancianos al ubicarlos en un lugar de pasividad.

La infantilización de los ancianos resulta una práctica habitual en este tipo de organizaciones y expresa claramente la idiosincrasia general en la que se inscribe. Respecto del modo en que los propietarios perciben a la familia podemos destacar la presencia de prejuicios, dado que los definen como irresponsables o abandónicos por el hecho mismo de haber internado a sus padres. Si bien muchas de las denuncias de este tipo tienen bases suficientes, en otros casos terminan constituyendo verdaderas profecías que se autocumplen, ya que es habitual que se excluya al fa- 
miliar de muchas de las decisiones sobre las rutinas cotidianas del anciano. En este contexto la institución termina sosteniendo el sentimiento de culpa que experimenta el familiar cuando decide internar al anciano, sentimiento que permanece gracias al refuerzo que le proveen las actitudes de los propietarios.

En el segundo aspecto se incluyen las cuestiones relacionadas con las características del servicio, el trato con el personal de la institución y las normas de funcionamiento.

En primer lugar, es necesario tener en cuenta que se trata de servicios privados en donde la rentabilidad suele ser el objetivo organizacional principal. Sin embargo muchos de los entrevistados incluso resaltan que su prioridad en el inicio de la actividad no es la económica.

El tamaño de las empresas suele ser reducido (en su mayoría la capacidad de atención no supera las treinta plazas), constituyendo un factor estratégico fundamental en el caso de los establecimientos geriátricos. Nótese que en su mayoría adoptan denominaciones que refieren al ámbito familiar y religioso: "Mi casa", "Casagrande", "En Familia", "El Hogar de los abuelos"," Madre Teresa de Calcuta".

El tamaño reducido permite establecer relaciones directas entre los usuarios y los miembros de la institución, donde al posibilitar una comunicación mas fluida, se produce un reconocimiento particularizado de las expectativas mutuas dando como resultado un alto grado de atención personalizada. En esta tesitura, debería pensarse que estas organizaciones pueden consolidar fácilmente el sentido de pertenencia y participación. Sin embargo esto no sucede debido a varios factores antes mencionados.

Cuando los entrevistados se refieren al personal es destacable que aparezcan modalidades de relación similares a las que se establecen entre el personal con los ancianos, especialmente en cuanto a la necesidad de controlar sus actividades. Los empleados suelen ser denominados con términos generalizadores como " las chicas", "mamitas", "las tías", etc, generalmente con alusiones de familiaridad como si tratara de personas con las que se ha convivido toda la vida.

Por otra parte respecto al perfil de empleado buscado por los propietarios se destacan mas características de personalidad que formación académica especializada, incluso en los casos en donde los mismos propietarios son médicos. El aspecto central que tienen en cuenta a la hora de contratar personal es la actitud hacia el anciano, menospreciando el nivel de capacita- ción sobre el tema, ya que se supone que la vocación supera al conocimiento y que en el mejor de los casos, lo que se necesita aprender se aprende haciéndolo. Es así como es frecuente la alusión al amor por los ancianos, el carisma, los dones especiales, etc. y al adiestramiento del personal en el inicio de su actividad en el establecimiento.

En cuanto al último aspecto resaltado en las entrevistas hemos mencionado los aspectos personales, en donde incluimos los motivos por los cuales la persona desarrolla esta actividad. La gerontología es en muchos casos un área residual donde terminan trabajando aquellos que no consiguen trabajo y no una orientación primaria (Muchinick, 1984) Aquí es posible observar como los intereses económicos superan a los vocacionales tan exaltados a lo largo de los relatos:

(...) "Me costó un montón arrancar porque viste sin medios, tenía un poquito de plata ahorrada y me alquilé una casa" (...), (...) "Siempre quise tener lo más lindo, y sigo pensando que lo voy a poner más lindo" (...), (...) "para mí no estoy equivocada, yo quiero a la persona verla, tratarla, tenerla, no soy perfecta pero yo quiero darle amor más bien, yo vivo de esto sí vivo, pero también ellos me están pagando para que yo les dé algo" (...), (...) "Y E. también pero la conocía acá, hacía una semana que estaba acá, bueno vino y ella por su propia decisión, porque quería vender el departamento, no quería estar sola, 90 años tiene, pero anda de acá para allá, vendió su departamento... yo en esto no quiero meterme en asunto de plata, no me vengas con asunto de plata, ahí no me encontras, no porque no quiero, por ahí ella tiene una hija y que se yo, ella no tiene hijos pero, eso si no me gusta, asuntos de plata...ella vendía y venía, yo no se ni cuanto lo vendió, ni donde, nada. No me gusta, no me interesa, yo estoy en otra cosa" (...).

Por otra parte como hemos mencionado anteriormente lo vocacional aparece resaltado en las motivaciones de los propietarios y encárgados. En dichas motivaciones es posible observar la presencia de actitudes anticipatorias y reparatorias. Las primeras aluden al la necesidad de ser cuidado en la vejez del mismo modo en que uno ha cuidado al anciano, y las reparatorias al hecho de cuidar al anciano por lo que no se ha cuidado a otros. (Muchinick, 1984)

\section{Conclusiones}

La persona que ingresa a una institución geriátrica deja de tener un estilo de vida independiente pasando a 
depender de un extraño que provee los cuidados. Por otra parte no se puede cuidar en masa ni en grupo porque cada ser humano tiene su propia realidad, su propio mundo y acompañarle en su enfermedad, en su vulnerabilidad actual, es apostar por su singularidad. Cada cual percibe el dolor, la enfermedad, el fracaso y la angustia desde su perspectiva personal, por lo que es necesario comprenderla para proveer cuidados efectivos..

Se observa repetidamente el énfasis en la necesidad de afecto del anciano y no tanto en la de un cuidado profesional, en donde se integre lo racional y afectivo. Se apuesta a la experiencia , al amor y las buenas intenciones desmereciendo la capacitación especifica que requieren las intervenciones gerontológicas.

Siguiendo las manifestaciones explícitas de los entrevistados, se acuerda en que las instituciones geriátricas persiguen como objetivo primordial la rehabilitación del residente, en realidad este objetivo rara vez se cumple. Las instituciones muchas veces terminan convirtiéndose en depósitos de personas, confirmando la imagen generalizada que se tiene de estos lugares.

La ILE constituye un proceso en donde las diferentes perspectivas muchas veces se contraponen dificultando la consecución de los objetivos que persigue la práctica de cuidado permanente. A lo largo de la investigación se observa como a cada palabra de alguno de los entrevistados aparece su contrapartida de parte de otro. Muchas veces antes que diálogos aparecen soliloquios incapaces de conectarse. El cuidado es una modalidad comunicativa, consiste en escuchar, requiere de conocimientos científicos tanto como de actitudes y valores que privilegien el mejoramiento de la vida y el despliegue de las potencialidades mas allá de las incapacidades.

\section{Bibliografía}

Berruti M.B.: Internación geriátrica : la perspectiva del investigador. Revista Margen http//www.margen.org 2001.

Berruti, Buzeki, de los Reyes, Roose: Ancianidad en instituciones de larga estadía: responsabilidades y solidaridad. Jornadas Argentinas y latinoamericanas de Bioética, Asociacion Argentina de Bioética, Mar del Plata. 1999

Flórez Lozano et al : Psicopatología de los cuidadores habituales de ancianos. Revista Departamento de Medicina. Universidad de Oviedo, Barcelona, 1997

Muchinick E. :Hacia una nueva imagen de la vejez. Ed. de Belgrano, Buenos Aires, 1984

Oddone M.J.: El Tercer Sector y la Tercera Edad, Conjuntos: Sociedad Civil en Argentina. Consejo Asesor de la Sociedad Civil. Banco Interamericano de Desarrollo en Argentina, Buenos Aires, Edilab Editora, 1998 Taylor y Bogdan, Taylor S.y Bogdan R. : Introducción a los métodos cualitativos de investigación. Buenos Aires, Paidós, 1986.

Torralba i Roselló F.: Antropología del cuidar, Fundación Mapfre Medicina,España 1998 